\section{Enigma of Respiratory Carriage of Kingella kingae and Neisseria meningitidis in Young Jordanian Children}

Malak A. Khanfar ${ }^{1}$, Eman Badran², Basma Marrar ${ }^{3}$, Ekatherina Charvalos ${ }^{4}$, Asem A. Shehabi ${ }^{1}$

1 Department of Pathology-Microbiology and Forensic Medicine, The Jordan University, School of Medicine, Amman Jordan.

\section{Abstract}

Background: Kingella kingae and Neisseria meningitidis are Gramnegative bacteria, causing several life-threatening diseases and are considered to be opportunistic pathogens in the upper respiratory tract of healthy carriers. The detection of these both bacterial species is difficult in routine culture methods.

Objective: This study aimed to find the occurrence rate of $K$. kingae and $N$. meningitidis colonizing upper respiratory tract of young Jordanian children, and to determine the antimicrobial susceptibility profile of the isolates.

Methods: A total of 300 samples of throat and nasal swabs were collected from out-patient Jordanian children aged between 6 months and 5 years, who were admitted to the pediatric clinical departments at the Jordan University Hospital and Al-Bashir Hospital over the period October 2018 through January 2019. Samples were cultured for detection of $K$. kingae and Neisseria species, including N. meningitidis. Their suspected growth was identified and tested using microbiology culture methods and polymerase chain reaction (PCR) method. Additionally, DNA was extracted directly from one 100 samples and was investigated only for $K$. kingae using real- time PCR assay.

Results: This study showed the absence of $K$. kingae in all cultured samples. Neisseria species was detected in 21 (7\%) including one $N$. meningitidis isolate $(0.3 \%)$. The results of antibiotic susceptibility testing indicated the presence of a low percentage of Neisseria species isolates resistant to clindamycin, oxacillin and vancomycin, whereas all were susceptible (100\%) to levofloxacin and gentamycin, and fewer to ampicillin (90.6\%) and erythromycin (85.7\%), respectively.
2 Department of Pediatrics, School of Medicine, Jordan University Hospital, Amman, Jordan.

3 Al-Bashir Hospital, Ministry of Health, Amman, Jordan.

4 IASO Hospital, Kifissias 37-39, Athens Greece 15123.

Contact information:

Prof. Dr. Asem A. Shehabi; Prof. Dr. Eman Badran.

\section{$\equiv$ asashehabi2@gmail.com ashehabi@ju.edu.jo e.badran@ju.edu.jo}

